\title{
PWM Dimming with Minimum Switching Cycle
}

\author{
Haishi WANG ${ }^{1, a}$, Tianbao WANG ${ }^{1, b}$, Hanfei YANG ${ }^{2, c}, \mathrm{Ke} \mathrm{LI}^{1, \mathrm{~d}}$ \\ ${ }^{1}$ Collaborative Innovation Center of Integrated Computation and Chip Security, Chengdu University \\ of Information technology, Chengdu, 610225, China
}

${ }^{2}$ Department of Microelectronics, Sichuan University, Chengdu, 610041, China

aemail: whs@cuit.edu.cn, bemail: wangtianbao@cuit.edu.cn, cemail: anakinyang1984@gmail.com, 'email: like213171@139.com

Keywords: LED, Dimming, PWM, Minimum Time, Preset Time

\begin{abstract}
PWM dimming is widely used to adjust the luminance of lighting emitting diode (LED) due to the advantage of no LED shimming. When the dimming ratio is relatively small, the LED current maybe unable to increase from zero to a desired level and thus the LED may be undersupplied with electricity. This paper proposed a new PWM dimming technique having a minimum switching cycle. The LED driver utilizing this technique comprises a boost converter having a pulse width detect circuit. The pulse width detect circuit compares the pulse width of the PWM dimming signal with a preset time, if the pulse width of the dimming signal is greater than the preset time, the boost converter is controlled by the dimming signal; if the width of the dimming signal is smaller than the pre-set time, the booster converter keeps its on-time equaling to the pre-set time, so the boost converter could transfer enough energy to the output capacitor to supply the LED. The LED driver is fabricated in SMIC with 0.35 um Bi-CMOS technology, silicon tests show that the LED driver could adapt all range of pulse width of dimming signal, even for a micro-second level pulse width.
\end{abstract}

\section{Introduction}

Along with the development of electronic devices, lighting emitting diode (LED) is widely used for its high light intensity, high energy efficiency, long life and environment-friendly characteristics [1-4]. At present, there are two dimming methods applying to adjust the luminance of LED. One is silicon controlled rectifier (also called TRIAC) dimming [5-8] and the other is pulse width modulation (PWM) dimming [9-12]. Compared with TRIAC dimming, PWM dimming has an advantage of no LED flickering and thus is preferred by the industry [13-15].

To solve issues originated from small dimming ratio, a new PWM dimming technique with a minimum switching cycle is proposed in this paper. The LED driver utilizing the new dimming technique could adapt all range of pulse width of dimming signal, even for a micro-second level pulse width.

\section{Issues in PWM dimming}

Figure 1 illustrates a LED driver with PWM dimmer. The LED driver comprises a boost converter as power supply, 4 LED strings as loads and 4 LED current sink amplifiers to set load current. In order to decrease power dissipation of the LED driver with PWM dimmer, PWM dimming signal is used to enable both the LED sink amplifier and the boost converter. Therefore during the off-time of the LED strings, the boost converter is also shut off by the PWM dimming signal.

The boost converter comprises a primary power switch, an output inductor, an output capacitor and a rectifier. It receives an input voltage VIN, and converts it into an output voltage VOUT for supplying the 4 LED strings. The boost converter comprises a minimum LED current select circuit for selecting the minimum voltage on cathodes of the 4 LEDs strings as a minimum feedback voltage (MFB)and providing it on an output terminal; an error amplifier (EA) for amplifying the 
difference between the MFB and a reference voltage VREF and generating an output voltage (COMP) accordingly; a current sense amplifier for sensing the current flowing through the primary power switch and generating a current sensing signal accordingly; an oscillator for providing a clock signal and a slope compensation signal; an PWM comparator for comparing the output voltage of the error amplifier with the current sensing signal, and a control circuit for generating a driving signal to control the $\mathrm{ON}$ and OFF of the primary switch. The primary power switch is turned on when the rising edge of the clock signal is coming and is turned off when the current sensing signal reaches COMP.

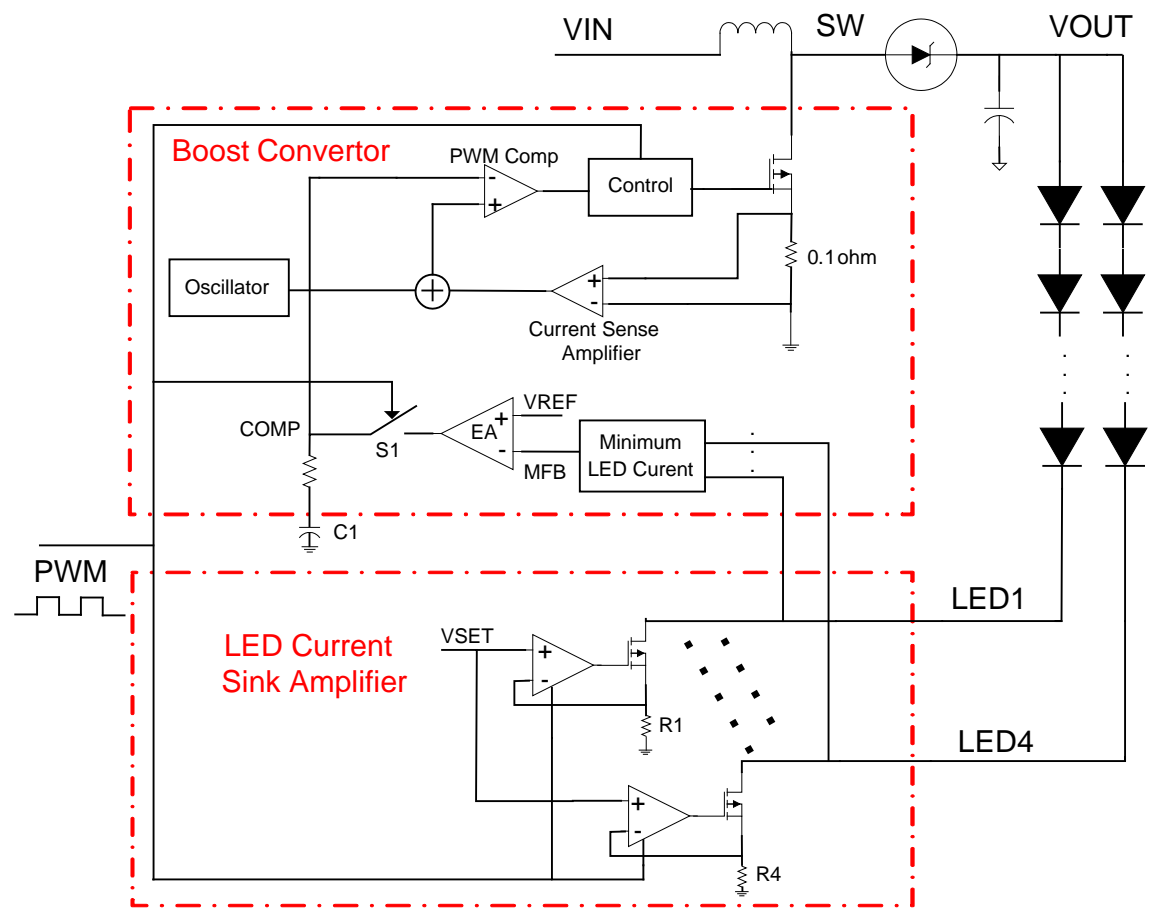

Fig.1. A LED driver with PWM dimming

Referring to figure 2(a) for dimming operation, at an initial state, when PWM dimming signal rises from logic low to logic high, the LED sink amplifier is enabled, and the boost converter begins to work, and the 4 LED strings are lightened. When PWM dimming signal falls from logic high to logic low, the LED sink amplifier is disabled and the 4 LED strings are shut down. To decrease the power dissipation and improve efficiency, the control circuit is also disabled when the PWM dimming signal is at logic low level.

However, when certain applications require for a wide range of dimming ratio, the dimming ratio (also called duty cycle) of the PWM dimming signal may be very small, and during one dimming cycle, the inductor current is even unable to increase from zero to a desired level. Thus the power supplied to the LED loads is not enough. If this situation maintains for a period, the output voltage of the converter will decrease and make the LED load function abnormally. As shown if figure 2(b), when the pulse width of the PWM dimming signal is around 10us, the peak LED current could just reach $20 \mathrm{~mA}$ while the rated LED current is about $300 \mathrm{~mA}$, and the output voltage of the boost converter continues decreasing.

\section{Proposed Dimming Technique}

To solve above issues of small dimming ratio, a new PWM dimming technique having a minimum switching cycle is proposed in this paper. Figure 3 illustrates a LED driver with proposed PWM dimming techniques. Compared with the LED driver shown in Figure 1, a pulse width detect circuit is integrated into the boost converter. The pulse width detect circuit is configured to compare the pulse width of the PWM dimming signal with a preset time and provide a dimming control signal (DCS) at an output terminal. If the pulse width of the dimming signal is greater than the pre-set time, the DCS is an exactly copy of the PWM dimming signal, and the proposed LED 
driver operate similarly as the LED driver shown in Figure 1. If the pulse width of the dimming signal is smaller than the pre-set time, the pulse width of the DCS is equal to the pre-set time. Since the boost converter is still keeps on at least for the pre-set time in each cycle, it could transfer enough energy to the output capacitor for supplying the LED. In this paper, the pre-set time is 4 times of the switching clock cycle of the boost converter.

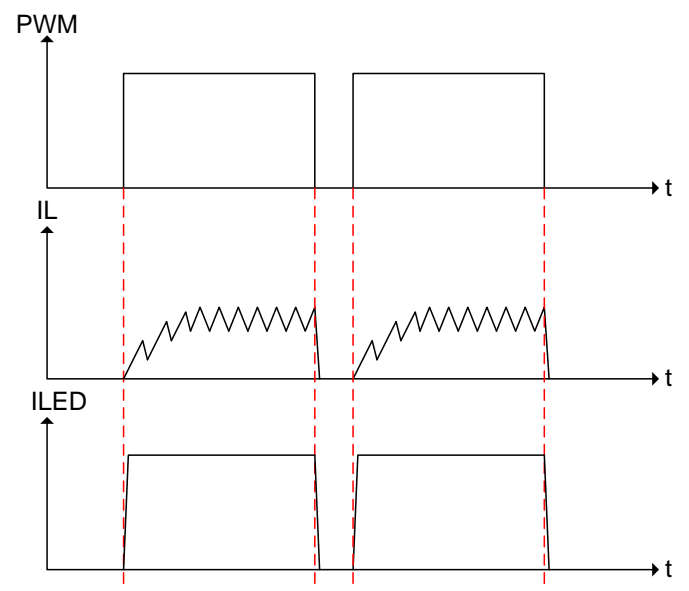

(a) Normal operation

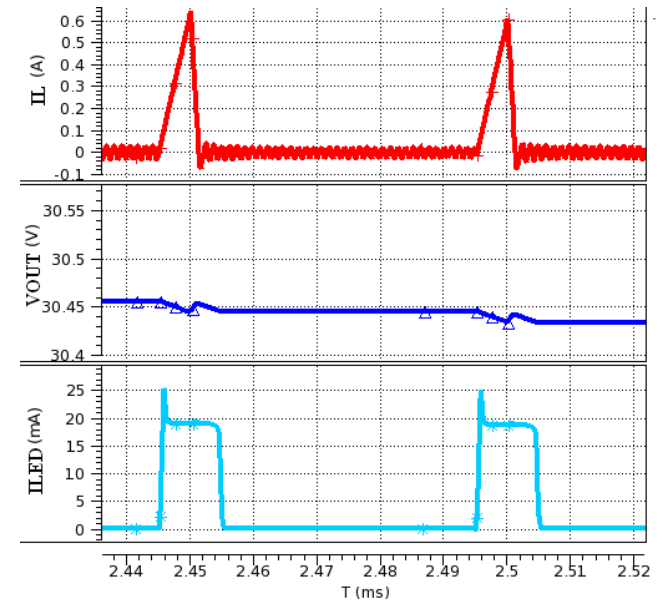

(b) Small dimming ratio

Figure.2. Traditional PWM dimming technique

Referring to Figure 3, the PWM dimming signal is compared with the clock signal generated by the oscillator. If the pulse width of the PWM dimming signal is greater than 4 times of the switching cycle, the width detect circuit provide the PWM dimming signal as its output directly. The DCS is exactly the copy of the PWM dimming signal, as shown in Figure 4(a). The proposed LED driver operate similarly as the LED driver shown in Figure 1, that is, when the PWM dimming signal is in logic high status, the boost converter and the LED sink amplifiers are turned on and the LED strings are lightened, and when the PWN dimming signal is in logic low status, the boost converter and the LED sink amplifiers are turned off, and there is no current flowing through LED strings.

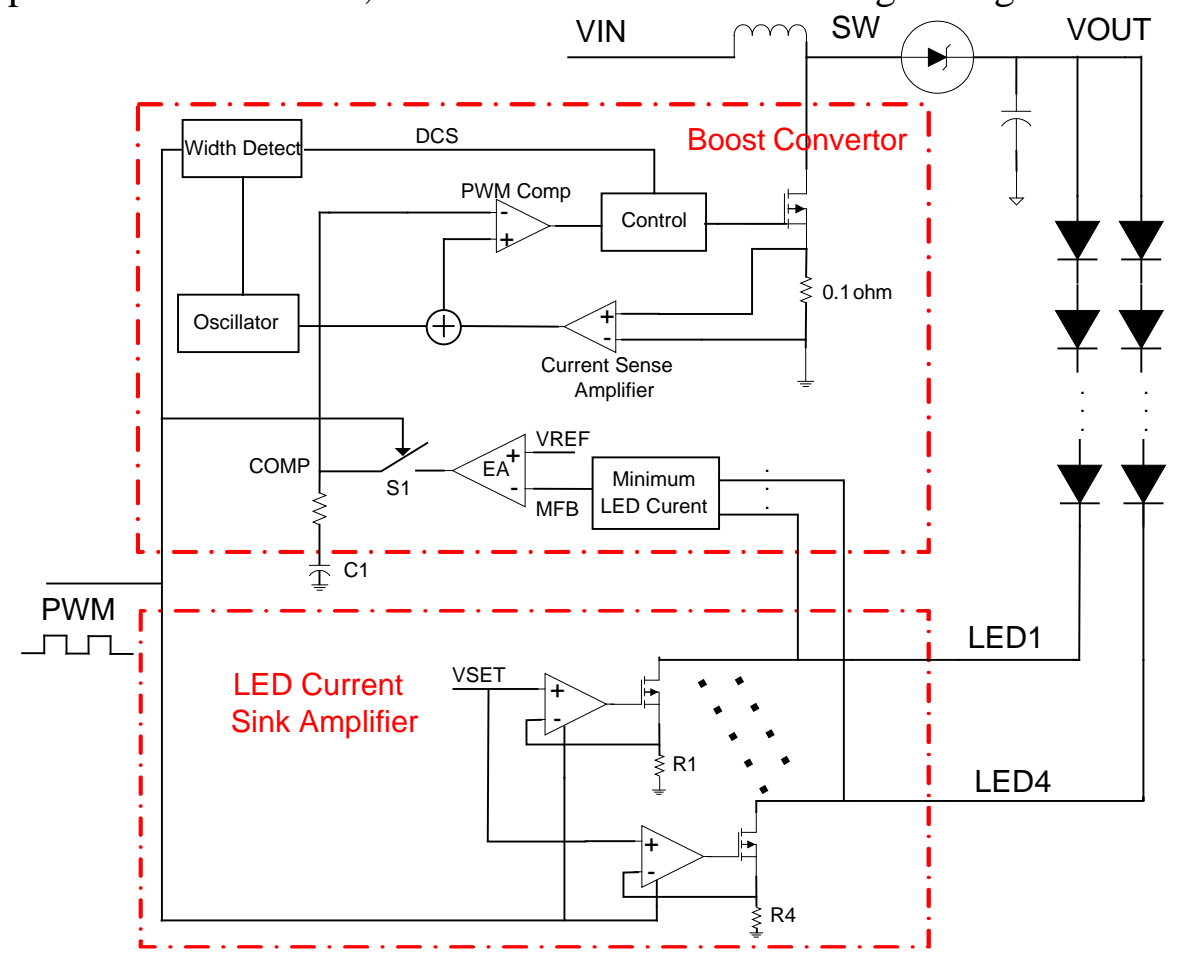

Figure.3. Proposed LED driver with minimum switching cycles

If the pulse width of the PWM dimming signal is smaller than 4 times of the switching cycle, the pulse width of DCS is maintained at 4 times of the switching cycle, as shown in Figure 4(b). In the first time when the PWM dimming signal is in high status, the boost and the LED sink amplifiers are turned on and the LED strings are lightened. When the PWM dimming signal falls from logic 
high to logic low, the switch S1 is turned off and the COMP is sustained due to the capacitor C1. LED sink amplifiers are disabled but the boost converter continues operating since the DCS is still at logic high, that is, the primary power switch keeps being turned on at the rising edge of the CLK signal and turned off when the current sensing signal reaches the constant COMP. The boost converter keeps operating until DCS falls to logic low.

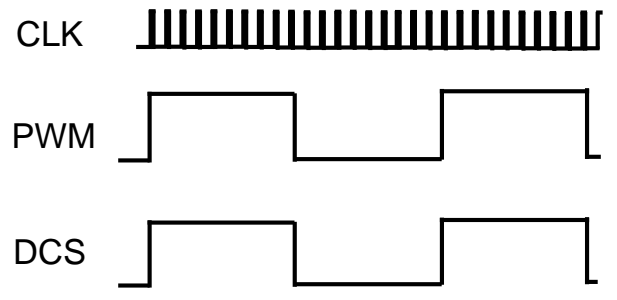

(a) Width $>4 \mathrm{X}$ clock cycles

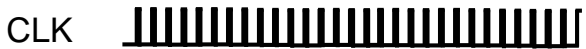

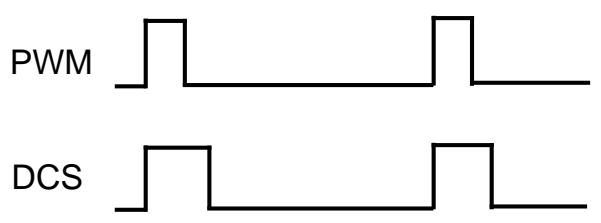

(b) Width $<4 \mathrm{X}$ clock cycles

Figure.4. Proposed PWM dimming technique

\section{Test results}

The proposed LED driver described above has been fabricated in SMIC with 0.35 um Bi-CMOS technology. Its layout and die micrograph are shown in Figure.5. The die size is about 2100umx1900um. The newly integrated pulse width detect circuit occupancies for only sever hundred square microns which could be neglected compared with the die size.

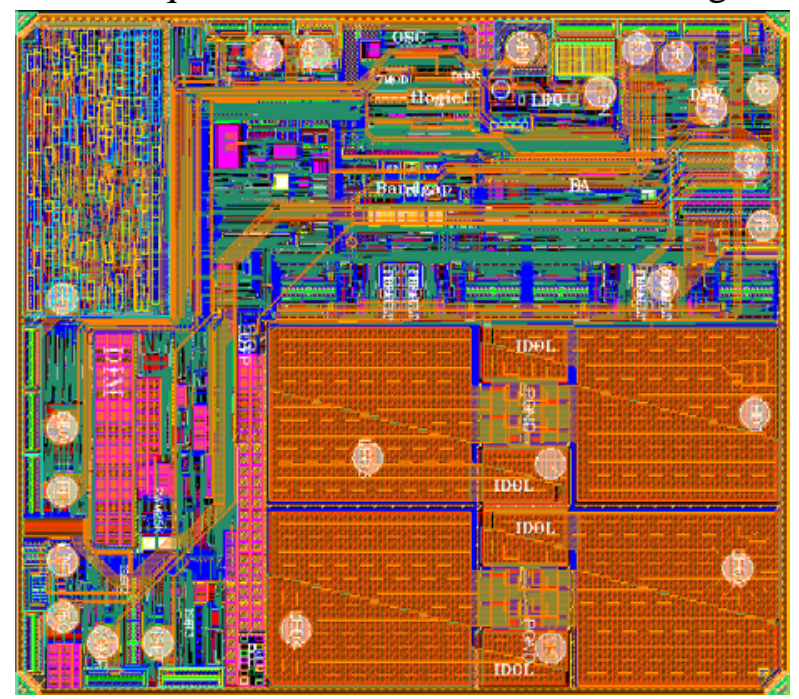

(a) Layout

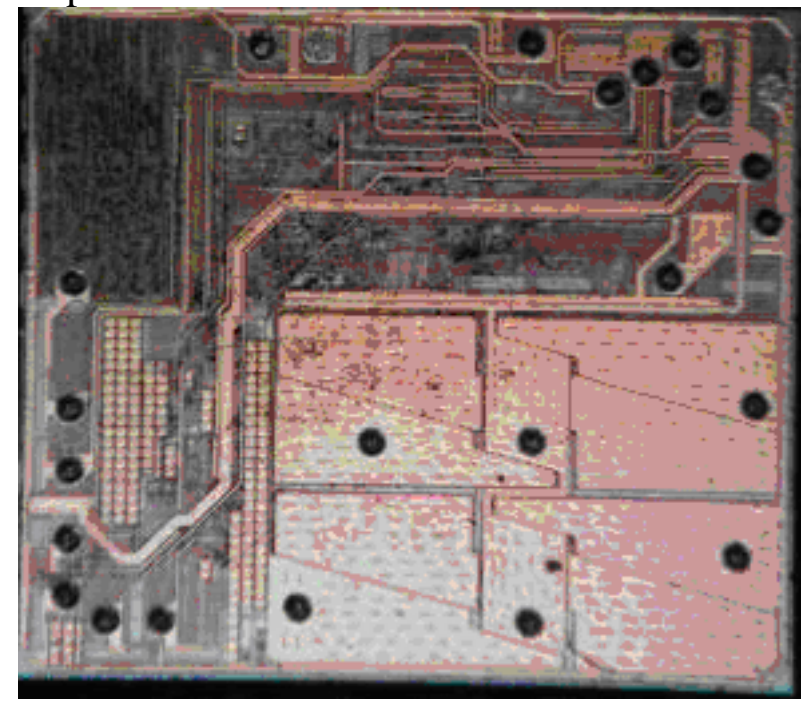

(b) Die micrograph

Figure.5. Micrographs of the proposed LED driver

Figure 6 shows operation waveforms with different dimming ratio. In Figure 7(a), the frequency of the PWM dimming signals is $2 \mathrm{KHz}$ and the duty cycle is $50 \%$. The waveforms of the proposed LED driver shown in Fig. 7(a) are similar with traditional LED drivers'. For those tests with small dimming ratio, the frequency of the PWM dimming signal is $2 \mathrm{KHz}$, but the pulse width is just about 2us which is smaller than the switching cycle 2.5us. As shown in Figure 7(b), the booster converter continues operating even the PWM dimming signal falls to logic low. Compared with Figure 2(b), the LED current shown in Figure 7(b) could reach the rated value and the VOUT are constant. 


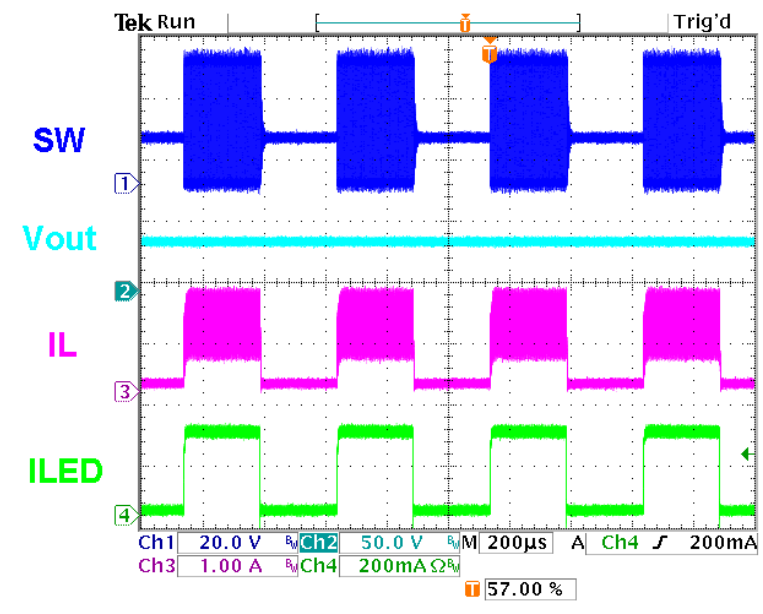

(a) Normal dimming ratio

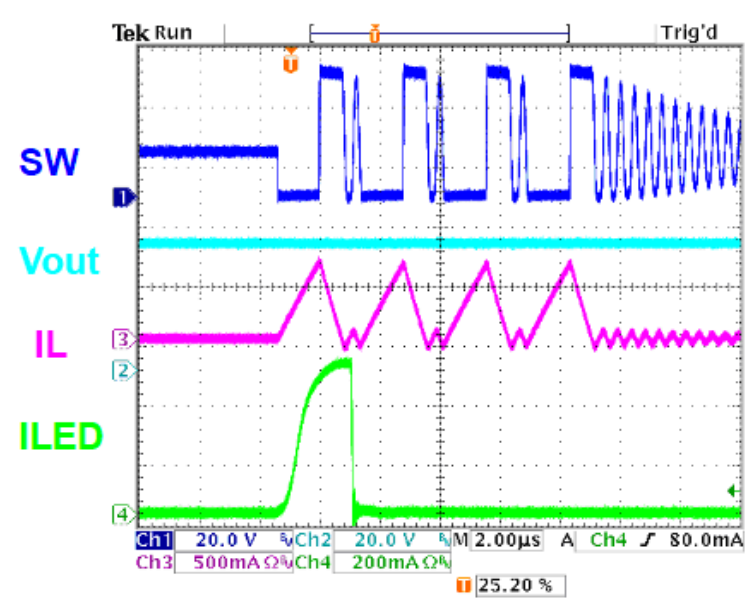

(b) Small dimming ratio

Figure.6. Test results in different dimming ratio

\section{Conclusion}

Based on analysis results to the root cause of dimming issues under small dimming ratio in traditional LED dimming techniques, this paper proposes a dimming technique having a minimum switching cycle. Silicon tests show that the proposed dimming technique has no negative effect to LED driver in normal dimming ratio and could provide sufficient energy to LED devices under small dimming ratio, even for a micro-second level pulse width. Both the layout occupation and the energy consummation of this technique are relatively small. Accordingly, this technique could be widely used in LED lighting systems.

\section{Acknowledgement}

The research was sponsored by the National Social Science Foundation (No. 61504014).

\section{References}

[1] Kyungnam Kang, Yongjeong Lee, Jungho Kim, et al. A Generalized Fabry-Pérot Formulation for Optical Modeling of Organic Light-Emitting Diodes Considering the Dipole Orientation and Light Polarization [J]. IEEE Photonics Journal, 2016(2)1600519.

[2] Hoang-Tuan Vu, Chun-Yuan Huang, Chih-Jung Chen, et al. An Efficient Material for Green Light-Emitting Diodes With Giant Quantum Dots [J]. IEEE Photonics Technology Letters, 2015(20)2123-2126.

[3] Huai Zheng, Jinlong Ma, Xiaobing Luo. Conformal Phosphor Distribution for White Lighting Emitting Diode Packaging by Conventional Dispensing Coating Method With Structure Control [J]. IEEE Transactions on Components, Packaging and Manufacturing Technology, 2013(3)417-421.

[4] Chia-Hung Wang, Tzu-Lun Hung, Tai-Ping Sun. PWM Photo-Power Control For Light-Emitting Diode Lighting System Without Photo-Diode Detection [C]. 2010 5th IEEE Conference on Industrial Electronics and Applications, 2010.1313-1316.

[5] SangCheol Moon, Gwan-Bon Koo, Gun-Woo Moon. Dimming-Feedback Control Method for TRIAC Dimmable LED Drivers [J]. IEEE Transactions on Industrial Electronics, 2015(2)960-965.

[6] Eun S. Lee, Jun P. Cheon, Duy N. Tan, et al. A novel TRIAC dimming LED driver by variable switched capacitance for power regulation [J]. 2014 IEEE Energy Conversion Congress and Exposition (ECCE), 2014.2600-2605.

[7] Weizhong Ma, Xiaogao Xie, Yang Han,et al. Control Scheme For Triac Dimming High Pf 
Single-Stage Led Driver With Adaptive Bleeder Circuit And Non-Linear Current Reference[C]. 2016 IEEE Applied Power Electronics Conference and Exposition (APEC), 2016.816-821.

[8] Eun S. Lee, Bo H. Choi, Duy T. Nguyen, Chun T. Rim. The Analysis Of Triac Dimming Led Driver By Variable Switched Capacitor For Long Life And High Power-Efficient Applications[C]. 2015 9th International Conference on Power Electronics and ECCE Asia (ICPE-ECCE Asia), 2015. 54-59.

[9] Brad Lehman, Arnold J. Wilkins. Designing to Mitigate Effects of Flicker in LED Lighting: Reducing risks to health and safety [J]. IEEE Power Electronics Magazine, 2014(3)18-26.

[10] Ali Mirvakili, Valencia J. Koomson, Michael Rahaim, et al. Wireless Access Test-Bed Through Visible Light And Dimming Compatible OFDM[C]. 2015 IEEE Wireless Communications and Networking Conference (WCNC), 2015. 2268-2272.

[11] Yong-Nong Chang, Hung-Liang Cheng, Chun-An Cheng, et al. An Lfpwm Dimmed Led Driver Featuring High Power Factor [C]. 2015 IEEE International Conference on Industrial technology (ICIT), 2015.914-919.

[12] Yan-Ming Li, Qian Tong, Xiao-Bing Yang, et al. Fixed-Frequency Adaptive Off-Time Controlled Buck Current Regulator With Excellent Pulse-Width Modulation And Analogue Dimming For Light-Emitting Diode Driving Applications [J]. IET Power Electronics, 2015(8) 2229 $-2236$.

[13] Zhenhua Yu, Robert J. Baxley, G. Tong Zhou. Brightness Control In Dynamic Range Constrained Visible Light Ofdm Systems[J]. 2014 23rd Wireless and Optical Communication Conference (WOCC), 2014.1-5.

[14] Yi-Tsung Chang, Ren-Hao Xue, Tzu-Chiao Sung, et al. Light Emitting Diode Constant-Current Driver Design Using By Pulse-Width Modulation Dimming Control[C]. 2015 IEEE 2nd International Future Energy Electronics Conference (IFEEC), 2015.1-5.

[15] Zhijiang Yang. Control Method for small PWM Duty Cycle Dimming of LED Driver [P]. US Patent No. 9265110, 2016.1-16. 